\title{
Criterial noise effects on rule-based category learning: The impact of delayed feedback
}

\author{
SHAWn W. ElL \\ University of Maine, Orono, Maine \\ AND \\ A. David Ing and W. Todd Maddox \\ University of Texas, Austin, Texas
}

\begin{abstract}
Variability in the representation of the decision criterion is assumed in many category-learning models, yet few studies have directly examined its impact. On each trial, criterial noise should result in drift in the criterion and will negatively impact categorization accuracy, particularly in rule-based categorization tasks, where learning depends on the maintenance and manipulation of decision criteria. In three experiments, we tested this hypothesis and examined the impact of working memory on slowing the drift rate. In Experiment 1, we examined the effect of drift by inserting a 5-sec delay between the categorization response and the delivery of corrective feedback, and working memory demand was manipulated by varying the number of decision criteria to be learned. Delayed feedback adversely affected performance, but only when working memory demand was high. In Experiment 2, we built on a classic finding in the absolute identification literature and demonstrated that distributing the criteria across multiple dimensions decreases the impact of drift during the delay. In Experiment 3, we confirmed that the effect of drift during the delay is moderated by working memory. These results provide important insights into the interplay between criterial noise and working memory, as well as providing important constraints for models of rule-based category learning.
\end{abstract}

Category learning is the process of establishing the memory traces necessary to organize objects and events in the environment into separate classes. Researchers have long debated the existence and qualitative nature of various category-learning systems. If any consensus has emerged from this debate, it is that categorization can occur by a process of explicit hypothesis testing (Allen \& Brooks, 1991; Ashby, Alfonso-Reese, Turken, \& Waldron, 1998; Erickson \& Kruschke, 1998; Folstein \& Van Petten, 2004; Nosofsky, Palmeri, \& McKinley, 1994; Regehr \& Brooks, 1995). According to the multiple learning systems perspective, a hypothesis-testing system would not be ideally suited for all category-learning tasks. Instead, a hypothesis-testing system is thought to be primarily responsible for learning rule-based tasks where accuracy is maximized by first learning which stimulus dimensions are relevant and then learning the placement of decision criteria along the relevant dimensions (Ashby et al., 1998; Ashby \& Ell, 2001). Not surprising, computational models implementing a hypothesis-testing system have focused on how the decision criteria are updated in response to trial-by-trial information (e.g., the stimuli, corrective feedback; Ashby et al., 1998; Busemeyer \& Myung, 1992; Erickson \& Kruschke, 1998; Kac, 1962; Kubovy \& Healy, 1977; Maddox, 2002; E. A. C. Thomas, 1973).
Many models of categorization and decision making also emphasize the effect of variability in the representation of the decision criterion, or criterial noise (e.g., Ashby et al., 1998; Ashby \& Lee, 1993; Benjamin, Diaz, \& Wee, 2009; Dorfman, Saslow, \& Simpson, 1975; Erev, 1998; Mueller \& Weidemann, 2008; Treisman \& Williams, 1984). Although criterial noise is thought to have a negative effect on categorization accuracy, few studies have examined how criterial noise effects might interact with the demands of rule-based category-learning tasks.

To begin, consider the categories in Figure 1A. Each point represents the spatial frequency and spatial orientation of a Gabor pattern (i.e., a sine wave grating in which contrast is modulated by a circular Gaussian filter). The optimal strategy involves learning the single unidimensional (1UD) decision criterion denoted by the solid vertical line. On each trial, the participant is presented with a single Gabor pattern and is instructed to assign the stimulus to Category A or B. Corrective feedback is provided immediately following the response, and the participant uses this feedback to learn the correct category assignments through trial and error. Thus, to learn this rulebased task, the participant needs to learn the placement of the most accurate (i.e., optimal) decision criterion on the spatial frequency dimension (i.e., the vertical lines).

S.W. Ell, shawn.ell@umit.maine.edu 


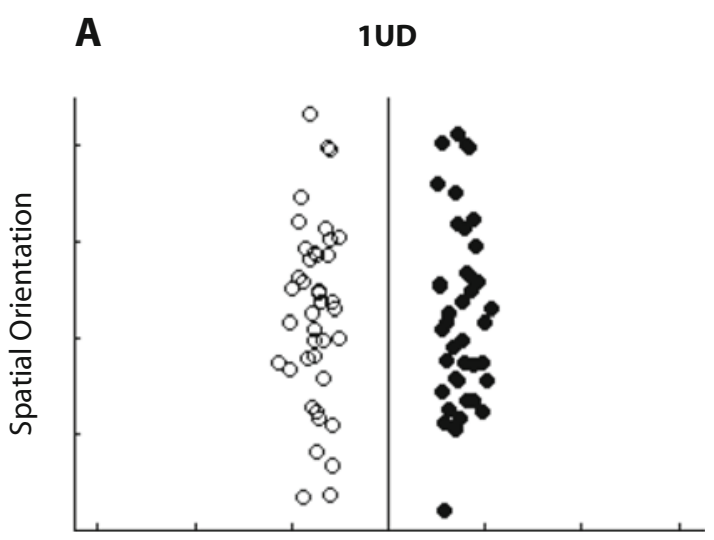

Spatial Frequency

B

2UD

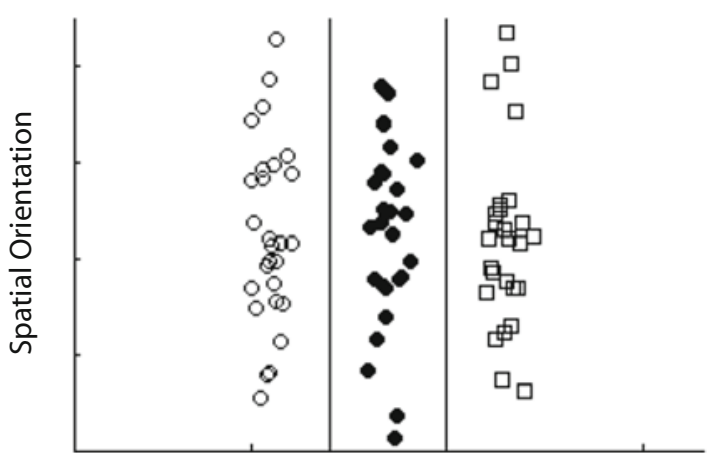

Spatial Frequency

\section{C}

3UD

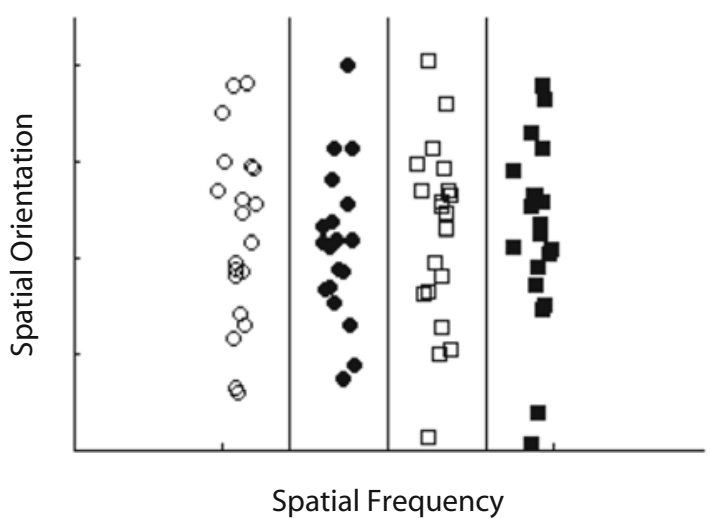

Figure 1. Category structures with (A) one, (B) two, or (C) three unidimensional (1UD, 2UD, and 3UD, respectively) decision criteria on the spatial frequency dimension. Each open circle denotes the spatial frequency and spatial orientation of a Gabor pattern from Category A. Each filled circle denotes a Gabor pattern from Category $B$. Each open square denotes a Gabor pattern from Category $C$. Each filled square denotes a Gabor pattern from Category $D$.
For the category structures in Figures 1B and 1C, learning proceeds in a similar fashion, with the exception that criterial learning demands are increased, because, in Figure $1 \mathrm{~B}$, two unidimensional (2UD) decision criteria and, in Figure 1C, three unidimensional (3UD) decision criteria must be learned. Criterial noise, as well as variability in the representation of the stimulus (i.e., perceptual noise) will affect learning, but our emphasis will be on criterial noise (we will return to the issue of criterial versus perceptual noise in the Discussion section).

Suppose that the representation of the decision criterion in Figure 1A is noisy and modeled by a zero-mean diffusion process (e.g., Ashby, 2000; Ratcliff, 1978). This implies that, with time, the representation of the decision criterion will naturally drift away from its mean position, thereby slowing the learning rate. Including additional decision criteria, as in Figures 1B and 1C, should increase the impact of drift on categorization accuracy. To verify this intuition, we developed and simulated the behavior of a model that used the optimal decision criteria (i.e., the solid lines in Figures 1A-1C). The goal of these simulations was to use a relatively simple model to provide a context in which to compare the impact of drift on categorization accuracy for the categories in Figures $1 \mathrm{~A}-1 \mathrm{C}$. Following previous approaches to modeling criterial noise (Erev, 1998; Mueller \& Weidemann, 2008), drift was modeled by assuming that there is within-trial variability in the representation of the decision criteria, with increased variability implying increased drift. On each trial, $i$, of the simulation, decision criterion $x_{i}$ was randomly shifted, such that $x_{i}=x_{\mathrm{opt}}+e_{\mathrm{c}}$, where $x_{\mathrm{opt}}$ is the position of the optimal decision criterion and $e_{\mathrm{c}}$ is criterial noise. The criterial noise, $e_{\mathrm{c}}$, was modeled as a stochastic process with $M=0$ and variance $=t \sigma^{2}$, where $t$ is time (in seconds; e.g., Cox \& Miller, 1965). The amount of drift in the decision criterion is determined by $\sigma$ and $t$, with smaller values of $\sigma$ resulting in a lower drift rate and smaller values of $t$ resulting in lower overall drift. Accuracy predictions were generated by averaging over 1,000 replications for $1 \leq \sigma \leq 5$ and $t=1$ and are plotted in Figure 2A. When the drift rate is low (approximately $\sigma \leq 2.5$ ), there is little impact of the number of decision criteria (one, two, or three) on accuracy. As the drift rate increases, however, increasing the number of decision criteria has an increasingly negative impact on accuracy (i.e., the 2UD and 3UD categories).

The duration of events in a typical rule-based categorylearning task (i.e., stimulus presentation, response, feedback, intertrial interval [ITI]) is often too short for the impact of drift in the decision criterion to be observable. One solution to this problem is to insert a delay during the normal sequence of events. For instance, delaying the delivery of corrective feedback following a response would be expected to result in poorer learning of the decision criterion. We replicated the simulations, letting $t$ represent the delay between the response and the feedback and increasing $t$ to $5 \mathrm{sec}$. As expected, accuracy decreased for all three category structures when the delay was increased (Figure 2B). As can be seen in Figure 2C, however, the impact of increasing the delay on accuracy was positively 


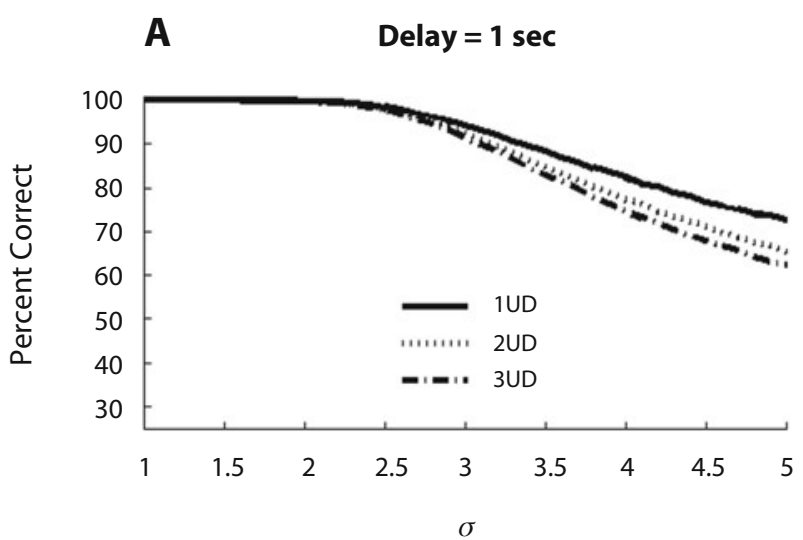

B Delay $=5 \mathrm{sec}$

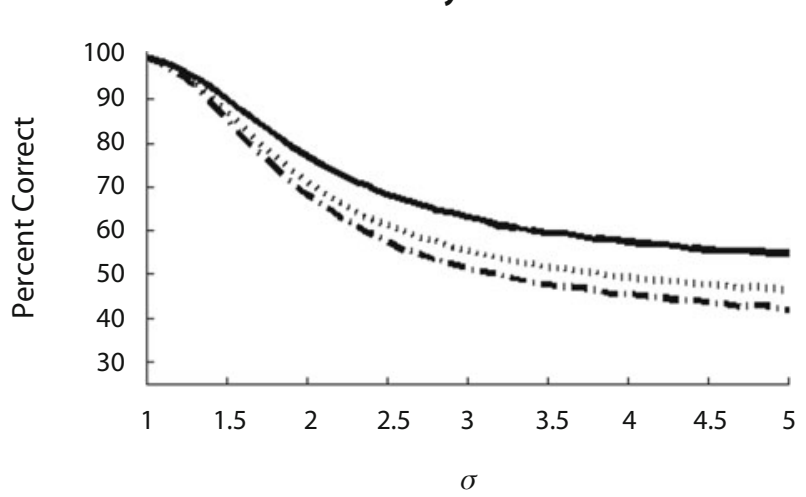

C Cost of Increasing Delay

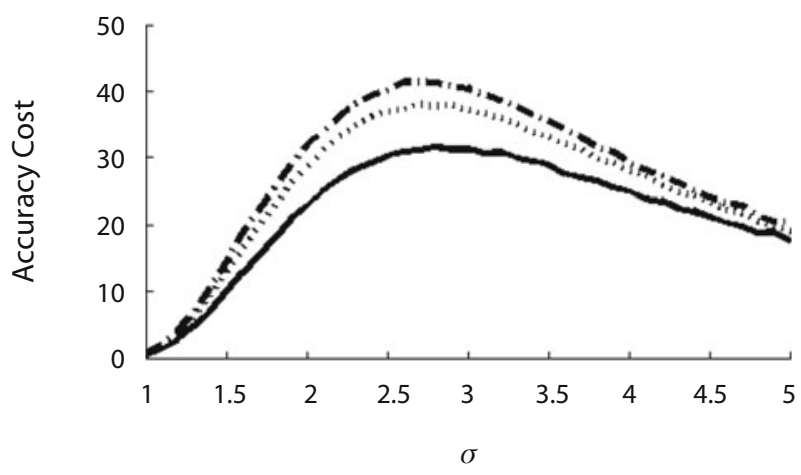

Figure 2. Accuracy predictions as a function of drift rate ( $\sigma$, the standard deviation of the criterial noise distribution) for the ideal observer simulations for the three category structures in Figure 1 (1UD, 2UD, and 3UD). (A) Predicted accuracy for a 1-sec delay (i.e., $t=1$ ). (B) Predicted accuracy for a 5-sec delay (i.e., $t=5$ ). (C) Accuracy cost of increasing the delay (predicted accuracy for delay $=1 \mathrm{sec}$ minus the predicted accuracy for delay $=5 \mathrm{sec}$ ).

correlated with the number of decision criteria, with the accuracy cost being greatest for the 3UD categories.

Although the accuracy cost is generally higher for the 3UD categories, it should be noted that an accuracy cost is predicted for most values of $\sigma$ for the 1UD and 2UD categories as well. Delaying feedback, however, by as long as $10 \mathrm{sec}$ when a single decision criterion is learned (i.e., similar to the 1UD categories in Figure 1A) does not result in an accuracy cost (Maddox, Ashby, \& Bohil, 2003). The most likely reason for the misprediction is the overly simplified model used in the simulations. For instance, given that rule-based category learning is highly dependent on working memory (i.e., the active maintenance and manipulation of the decision criteria; Ashby \& Ell, 2002; Maddox, Ashby, Ing, \& Pickering, 2004; Maddox, Filoteo, Hejl, \& Ing, 2004; Waldron \& Ashby, 2001; Zeithamova \& Maddox, 2006, 2007), it is reasonable to assume that working memory could slow the drift by stabilizing the representation of the decision criterion. ${ }^{1}$

Interestingly, the drift rates in the simulations in Figures $2 \mathrm{~A}-2 \mathrm{C}$ that predict small accuracy costs for 1UD also predict no accuracy cost as the number of criteria increases. Thus, it may be that working memory resources are sufficient to stabilize the drift regardless of the number of decision criteria. An alternative hypothesis, however, is that working memory can slow the drift rate but, because working memory has a limited capacity, the system's efficiency will decrease as the number of decision criteria to be represented increases. Thus, the impact of delayed feedback on accuracy should increase dramatically once the demands of the task exceed working memory capacity limitations. Given that there was no effect of delaying feedback when participants were required to learn a single decision criterion (Maddox et al., 2003), we opted to investigate this hypothesis by focusing on rule-based category-learning tasks with two (2UD) and three (3UD) decision criteria.

\section{EXPERIMENT 1 Method}

\section{Participants and Design}

A total of 105 participants were solicited from the University of Texas community and received course credit for participation. The participants were randomly assigned to the six experimental conditions as follows: 2UD delay $(n=24), 2 \mathrm{UD}$ immediate $(n=26)$, 3UD delay $(n=24)$, and 3UD immediate $(n=31)$. No participant completed more than one experimental condition, and each session lasted approximately $60 \mathrm{~min}$. All of the participants were tested for 20/20 vision using a Snellen eye chart. In nearly all of our current work with two categories, we define a learner as a participant who achieves $65 \%$ accuracy during the final block of trials. To account for lower chance accuracy due to the increased number of categories, we lowered the criterion proportionally to $43 \%$ accuracy and $32.5 \%$ accuracy during the final block of trials for the two (2UD) decision criteria and three (3UD) decision criteria conditions, respectively. The data from participants who did not meet this criterion were excluded from all subsequent analyses. These criteria resulted in the following exclusions: 2UD delay $(n=6), 2 \mathrm{UD}$ immediate $(n=4)$, 3UD delay $(n=5)$, 3UD immediate $(n=6)$.

\section{Stimuli and Stimulus Generation}

In Experiment 1, we used the randomization technique introduced by Ashby and Gott (1988). The stimuli (2UD, 81 total, 27 from each of the three categories; 3UD, 80 total, 20 from each of the four categories) were generated by sampling randomly from separate bivariate normal distributions (see Table 1 for category parameters). One set of stimuli was generated and the presentation order was randomized in each of four blocks of trials for every participant.

The stimuli were computer generated and displayed on a 21-in. monitor with $1,360 \times 1,024$ pixel resolution in a dimly lit room. 
Table 1

Category Distribution Parameters

\begin{tabular}{|c|c|c|c|c|c|c|}
\hline Condition & Category & $\mu_{f}$ & $\mu_{o}$ & $\sigma_{f}^{2}$ & $\sigma_{o}^{2}$ & $\operatorname{cov}_{f, o}$ \\
\hline \multicolumn{7}{|c|}{ Experiment 1} \\
\hline \multirow[t]{3}{*}{ 2UD } & A & 255 & 125 & 9 & 9,000 & 0 \\
\hline & B & 285 & 125 & 9 & 9,000 & 0 \\
\hline & $\mathrm{C}$ & 315 & 125 & 9 & 9,000 & 0 \\
\hline \multirow[t]{4}{*}{$3 \mathrm{UD}$} & A & 255 & 125 & 9 & 9,000 & 0 \\
\hline & B & 285 & 125 & 9 & 9,000 & 0 \\
\hline & $\mathrm{C}$ & 315 & 125 & 9 & 9,000 & 0 \\
\hline & $\mathrm{D}$ & 345 & 125 & 9 & 9,000 & 0 \\
\hline \multicolumn{7}{|c|}{ Experiment 2} \\
\hline \multirow[t]{6}{*}{$4 \mathrm{CJ}$} & $A_{1}$ & 260 & 100 & 25 & 625 & 0 \\
\hline & $\mathrm{A}_{2}$ & 260 & 200 & 25 & 625 & 0 \\
\hline & $\mathrm{B}^{2}$ & 300 & 100 & 25 & 625 & 0 \\
\hline & $\mathrm{C}$ & 300 & 200 & 25 & 625 & 0 \\
\hline & $\mathrm{D}_{1}$ & 340 & 100 & 25 & 625 & 0 \\
\hline & $\mathrm{D}_{2}$ & 340 & 200 & 25 & 625 & 0 \\
\hline
\end{tabular}

Each Gabor pattern was generated using MATLAB routines from the Psychophysics Toolbox (Brainard, 1997; Pelli, 1997). Each random sample $\left(x_{1}, x_{2}\right)$ was converted to a stimulus by deriving the frequency, $f=.25+\left(x_{1} / 50\right)$, and orientation, $o=x_{2}(\pi / 500)$. For example, the Category A mean for the 2UD category structure was converted to a Gabor pattern with frequency $f=.25+(255 / 50)=$ $5.35 \mathrm{cyc} / \mathrm{deg}$ and orientation $o=125(\pi / 500)=0.785$ radians counterclockwise from horizontal. The scaling factors were chosen in an attempt to equate the salience of frequency and orientation.

\section{Procedure}

The participants were informed of the number of categories and that each category was equally likely to appear. They were informed that perfect performance was possible and were instructed to learn about the categories. They were told to be as accurate as possible and not to worry about speed of responding. The procedure for a typical trial was as follows. The immediate feedback condition consisted of a response-terminated stimulus display, a $500-\mathrm{msec}$ mask, $750 \mathrm{msec}$ of feedback, and a 5-sec blank-screen ITI. The delayed feedback condition consisted of a response-terminated stimulus display, a 5-sec mask, $750 \mathrm{msec}$ of feedback, and a 500-msec blank-screen ITI.

The mask was a Gabor pattern that subtended approximately $11^{\circ}$ of visual angle and was of a random frequency and orientation from within the range of stimulus values.

\section{Results and Theoretical Analyses}

\section{Accuracy-Based Analyses}

Inspection of the average accuracy data in Figure 3 shows an accuracy cost when feedback was delayed, but only when three decision criteria were learned. To directly test this observation, we analyzed the effect of feedback delay for the 2UD and 3UD categories using separate 2 (feedback condition: immediate vs. delayed) $\times 4$ (block) mixed ANOVAs. For 2UD criteria, only the main effect of block was significant $\left[F(3,114)=58.73, M S_{\mathrm{e}}=.01\right.$, $p<.001]$, with both the main effect of feedback condition and the interaction yielding $F$ s less than 1 . For 3UD criteria, the main effects of block $\left[F(3,126)=66.02, M S_{\mathrm{e}}=\right.$ $.009, p<.001]$ and feedback condition $[F(1,42)=58.73$, $\left.M S_{\mathrm{e}}=.08, p<.05\right]$ were significant, and the interaction was marginally significant $\left[F(3,126)=2.40, M S_{\mathrm{e}}=.009\right.$, $p=.07]$.

These results are consistent with the hypothesis that delayed feedback increases the drift in the decision criteria and thus impairs accuracy, but only when the demands of the task exceed capacity limitations (i.e., the 3UD categories). The finding of no accuracy cost in the 2UD categories (and in the 1UD categories of Maddox et al., 2003) suggests that the effect of drift can be overcome, but only when the demands on working memory resources are sufficiently low. More specifically, we would argue that the working memory demand due to the requirement to manipulate and maintain three decision criteria when there is a 5-sec delay between response and feedback exceeds the working memory capacity needed to learn these rulebased tasks.

\section{Model-Based Analyses}

Following Maddox et al. (2003), we applied a series of decision-bound models to the data to determine the types of strategies that participants might use to solve these tasks. Given that observed accuracy was well below asymptote, we cannot assume that the participants were responding optimally. Thus, it is important to verify that the participants were using strategies that assume the representation of a decision criterion. Decision-bound models were fit separately to the data from each participant and each block. When it is informative, we provide information about of the all blocks, but, for brevity, we focus on the results from the final block of data.

Decision-bound models are derived from general recognition theory (GRT; Ashby \& Townsend, 1986), which is a multivariate generalization of signal detection theory (e.g., Green \& Swets, 1966). GRT assumes that there is trial-by-trial variability in the perceptual information obtained from every stimulus, no matter what the viewing conditions (Ashby \& Lee, 1993). GRT assumes that each participant partitions the perceptual space into response regions by constructing decision boundaries to separate the regions. On each trial, the participant determines which

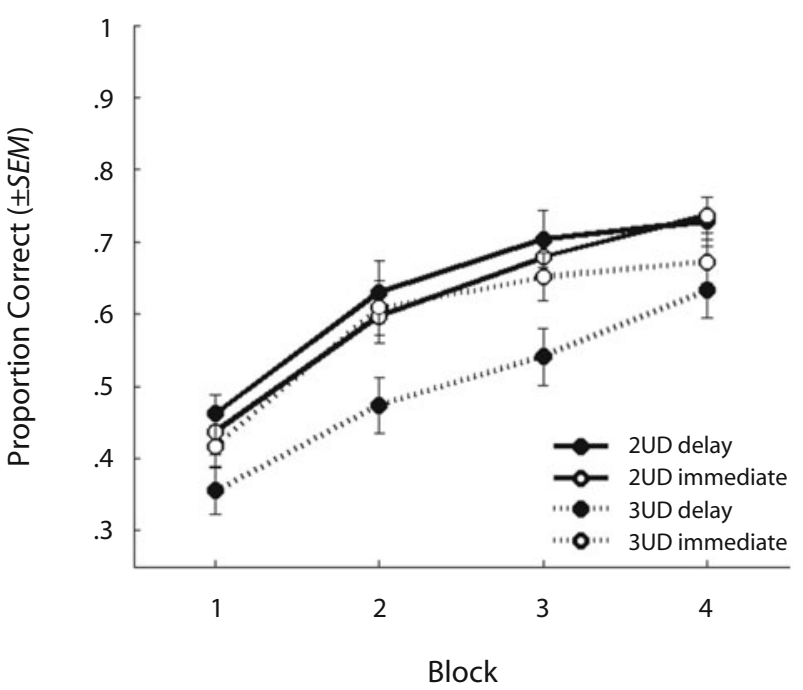

Figure 3. Proportion correct for the delayed- and immediatefeedback conditions of Experiment 1. 2UD, two-unidimensional condition; 3UD, three-unidimensional condition. 
region the percept is in and then emits the associated response. Despite this deterministic decision rule, decisionbound models predict probabilistic responding because of trial-by-trial variability that occurs as a result of perceptual and criterial noise. Two different classes of decisionbound models were fit to the data (see Ashby, 1992a, for a more formal treatment of these models; Maddox \& Ashby, 1993). One type is compatible with the assumption that the participants used an explicit hypothesis-testing strategy, and one type assumes an information-integration strategy.

Hypothesis-testing models. 2UD rule-based models were applied. The unidimensional, spatial frequency model assumes that the participant sets two (2UD categories) or three (3UD categories) criteria along the spatial frequency dimension. For the 2UD categories, the model uses the rule respond $A$ if the spatial frequency is low, respond $B$ if the spatial frequency is intermediate, respond $C$ if the spatial frequency is high. This model has three (2UD) or four (3UD) free parameters: one parameter for each decision criterion and one noise variance. A special case of this model assumes that participants use the optimal decision strategy (i.e., the vertical decision bounds in Figure 1B or 1C) with noise variance being the only free parameter.

Information-integration model. The minimum distance classifier assumes that there are three units, one associated with each category, in the frequency-orientation space. On each trial, the participant determines which unit is closest to the perceptual effect and gives the associated response. Because the location of one of the units can be fixed and since a uniform expansion or contraction of the space will not affect the location of the resulting (minimum distance) decision bounds, the model for the 2 UD categories contains four free parameters (i.e., three that determine the location of the units associated with Categories $\mathrm{A}-\mathrm{C}$ and one noise variance). The model for the 3UD categories includes an additional parameter determining the location of the unit associated with Category D. This model is assumed to make categorization decisions by combining information across the spatial frequency and orientation dimensions, rather than by setting decision criteria on the spatial frequency dimension, as is the case with the hypothesis-testing models (Ashby \& Waldron, 1999)

Model fits. The model parameters were estimated using maximum likelihood (Ashby, 1992b; Wickens, 1982), and the goodness-of-fit statistic was AIC $=2 r-2 \ln L$, where $r$ is the number of free parameters, and $L$ is the likelihood of the model given the data (Akaike, 1974; Takane \& Shibayama, 1992). The AIC statistic penalizes a model for extra free parameters in such a way that the smaller the AIC, the closer a model is to the true model, regardless of the number of free parameters. Thus, to find the best model among a given set of competitors, one simply computes an AIC value for each model and chooses the model associated with the smallest AIC value.

Using AIC, we determined which of the model typeshypothesis testing or information integration - provided the best account of the final block of data for each par- ticipant tested with the 2UD categories. We computed the percentage of responses accounted for by the best-fitting model and found relatively large values, suggesting that the model comparisons are valid (delayed: hypothesis testing, $73 \%, S D=15$; information integration, $72 \%, S D=17$; immediate: hypothesis testing, $72 \%, S D=15$; information integration, $82 \%, S D=4$ ). As predicted, the percentage of participants using hypothesis-testing strategies was high for both immediate- (82\%) and delayed-feedback (83\%) conditions, and the accuracy rate achieved by these participants was also high $(73 \%$ and $75 \%$ for the immediateand delayed-feedback conditions, respectively). Thus, the majority of participants were using hypothesis-testing strategies, and, consistent with the accuracy analyses, their performances were similar for the immediate- and delayed-feedback conditions.

For the 3UD categories, we fit the hypothesis-testing and information-integration models to each participant's data on a block-by-block basis. ${ }^{2}$ In the delayed-feedback condition, the percentage of observers using a hypothesistesting strategy was high in all four blocks $(90 \%, 78 \%$, $79 \%$, and $78 \%$ in Blocks 1-4, respectively). A similar pattern held in the immediate-feedback condition (84\%, $88 \%, 84 \%$, and $80 \%$ in Blocks $1-4$, respectively). On the other hand, the accuracy rates for these participants were lower in the delayed- than in the immediate-feedback condition in Blocks 1-3 (delayed, 34\%, $47 \%$, and $52 \%$ in Blocks 1-3, respectively; immediate, $43 \%, 59 \%$, and $64 \%$ in Blocks $1-3$, respectively) but were equivalent in Block 4 (67\% in both the immediate- and delayedfeedback conditions).

To identify the locus of the delayed-feedback effect on rule-based learning during the first three blocks of trials, we examined the parameter estimates from the suboptimal spatial frequency model. We computed the absolute deviation between the observed decision criteria and the optimal criteria for each of the three criteria and averaged those absolute deviations. Large absolute deviations are associated with highly suboptimal decision criterion placements and, thus, with poor learning, whereas small absolute deviations are associated with more nearly optimal decision criterion placement and, thus, with good learning. Focusing only on the participants whose data were best fit by a hypothesis-testing model, the median for the absolute deviation measure was 35.2, 9.7, and 12.9 for the delayedfeedback condition in Blocks 1-3, respectively, and was 17.7, 5.7, and 5.1 for the immediate-feedback condition in Blocks 1-3, respectively, suggesting that the decision criteria of participants in the delayed-feedback condition deviated more from optimal than did those of participants in the immediate-feedback condition.

In addition, we examined the noise standard deviation from the same model. The noise standard deviation is a measure of the memory and the consistent application of the learned decision criteria, as well as the memory of the perceptual representation of the stimulus (with lower values representing less criterial and perceptual noise). The median for the noise measure was 10.6, 6.8, and 5.7 for the delayed-feedback condition in Blocks 1-3, respectively, and was $8.2,4.7$, and 4.7 for the immediate- 
feedback condition in Blocks 1-3, respectively. Thus, the delayed feedback effect early in rule-based learning for the 3UD categories appears to be due primarily to poor learning of the placement of the decision criteria and to a slight increase in criterial and perceptual noise.

\section{EXPERIMENT 2}

The results of Experiment 1 support the hypothesis that the increase in working memory demand associated with learning three decision criteria leads to an accuracy cost in rule-based category learning. We would argue, however, that there is nothing special about three decision criteria. For instance, it may simply be more difficult to maintain the representation of three decision criteria defined along a single dimension, relative to distributing the criteria across multiple dimensions. Work in absolute identification - a closely related paradigm in which participants are asked to assign a unique response to each unique stimulus - shows that identification performance improves as the number of dimensions relevant to solving the task increases (Attneave, 1959; Garner, 1962; Miller, 1956; Pollack, 1952). For example, participants have great difficulty learning to identify nine lines of different lengths but have little difficulty learning nine lines constructed from the factorial combination of three line lengths with three line orientations. Miller hypothesized that the multidimensional benefit emerges because information transmission is increased (i.e., an increased correlation between the stimulus input and identification response) when identifying multidimensional, relative to unidimensional, stimuli. If the decision criteria are also considered to be input to the decision process, it seems reasonable to assume that the multidimensional benefit observed in absolute identification should generalize to rule-based category learning. Such a finding would also be consistent with research arguing that identification and categorization involve a similar decision process (Ashby \& Lee, 1991; Maddox, 2001, 2002; Nosofsky, 1986; Shepard, Hovland, \& Jenkins, 1961), given that the primary difference is in the nature of the stimulus-response mapping (many-to-one in category learning versus one-toone in identification).

Increased information transmission is likely to have many benefits for cognition, but we hypothesize that one indirect benefit would be reduced working memory demand. Thus, when the decision criteria are distributed across two dimensions, relative to all criteria being defined on a single dimension (i.e., the 3UD categories), working memory resources would be available to slow the drift rate on the representation of the decision criteria, resulting in a reduction in the effect of delayed feedback. We tested this prediction in Experiment 2 using the category structure in Figure 4. We refer to this as the 3CJ condition, since the decision rule requires a conjunction of three decision criteria across both spatial frequency and orientation. The optimal strategy requires the participant to set two criteria on spatial frequency and one on spatial orientation using the following rule: Respond $A$ if the frequency is low, respond $B$ if the frequency is intermediate and the orientation is shallow, respond $C$ if the frequency is intermediate and the orientation is steep, respond $D$ if the frequency is high. Because we want to compare performance directly between the 3UD condition from Experiment 1 and the 3CJ condition from Experiment 2, we equated immediate-feedback performance between the conditions. We did this by conducting a series of small pilot studies to determine the appropriate category distribution parameters to achieve this goal.

\section{Method}

\section{Participants and Design}

Fifty-six participants were solicited from the University of Texas community and received course credit for participation. The participants were randomly assigned to the two experimental conditions as follows: 3CJ delay $(n=28)$ and 3CJ immediate $(n=28)$. No participant completed more than one experimental condition, and each session lasted approximately $60 \mathrm{~min}$. All of the participants were tested for 20/20 vision using a Snellen eye chart. A learning criterion of $32.5 \%$ correct during the final block resulted in the following exclusions: delayed $(n=5)$ and immediate $(n=4)$.

\section{Stimuli and Stimulus Generation}

The stimuli, stimulus generation, and experimental procedures were identical to those in the 3UD condition of Experiment 1, with the exception that different parameters were used to generate the categories (see Table 1).

\section{Results and Theoretical Analyses}

\section{Accuracy-Based Analyses}

Average accuracy from the delayed- and immediatefeedback conditions for the 3CJ and 3UD (replotted for reference) conditions are displayed in Figure 5. As can be seen in Figure 5, there was no effect of delay on rule-based learning when the participant was required to learn two decision

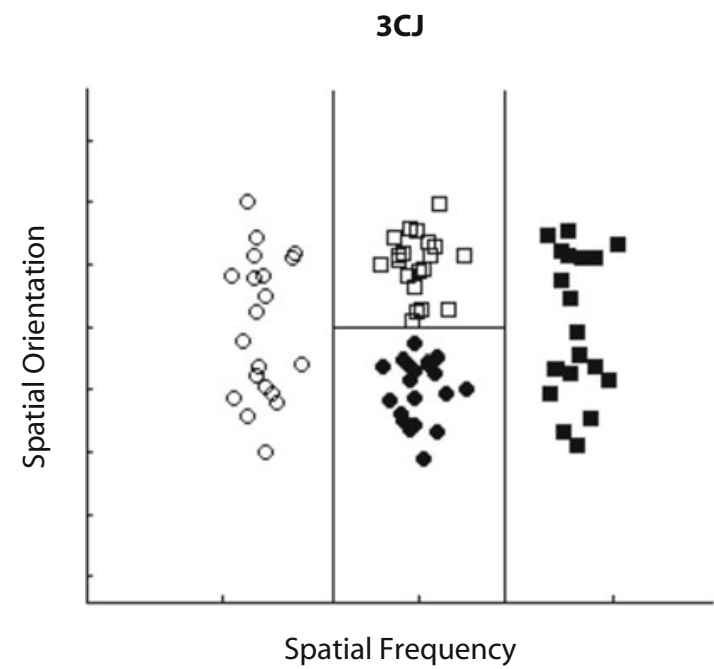

Figure 4. Category structure from Experiment 2. Each open circle denotes the spatial frequency and spatial orientation of a Gabor pattern from Category A. Each filled circle denotes a Gabor pattern from Category B. Each open square denotes a Gabor pattern from Category C. Each filled square denotes a Gabor pattern from Category D. 3CJ, three-decision-criteria conjunction condition. 
criteria along the frequency dimension and one along the orientation dimension (3CJ). To compare this result with that for the 3UD rule-based task, we must demonstrate that accuracy was equated in the immediate-feedback conditions. We demonstrate this by comparing performance on a block-by-block basis. In all four blocks, performance did not differ (all $p \mathrm{~s}>.05$ ), suggesting that we were successful at equating immediate-feedback performances across the two rule-based conditions. To determine whether delayed feedback affects $3 \mathrm{CJ}$ category learning, a 2 (feedback condition: immediate vs. delayed) $\times 4$ (block) mixed design ANOVA was conducted on the accuracy rates. Only the main effect of block $\left[F(3,135)=77.88, M S_{\mathrm{e}}=.010, p<\right.$ $.001]$ was significant, with both the main effect of feedback condition and the interaction yielding $F$ s less than 1 . As was the case in Experiment 1, the majority of the participants in the immediate- $(84 \%)$ and delayed- $(81 \%)$ feedback conditions were using hypothesis-testing strategies during the final block. The results of the analysis of the accuracy data were unchanged when only those participants were included that were best fit by a hypothesis-testing strategy.

The interpretation of Experiment 2 depends on the assumption that the accuracy rates were equated across the 3UD and 3CJ immediate-feedback conditions. Accuracies did not differ significantly, but 3UD accuracy was numerically higher than $3 \mathrm{CJ}$ accuracy. Thus, a task-difficulty hypothesis would predict a larger delayed-feedback effect for the 3CJ categories, which is the opposite of the effect observed. Instead, the delayed-feedback effect was observed in the easier (i.e., 3UD) task. Thus, consistent with the predictions based on the absolute identification literature, the absolute number of decision criteria is not the critical factor in determining whether drift in the decision criteria will result in an accuracy cost when feedback is delayed. Instead, these data are consistent with

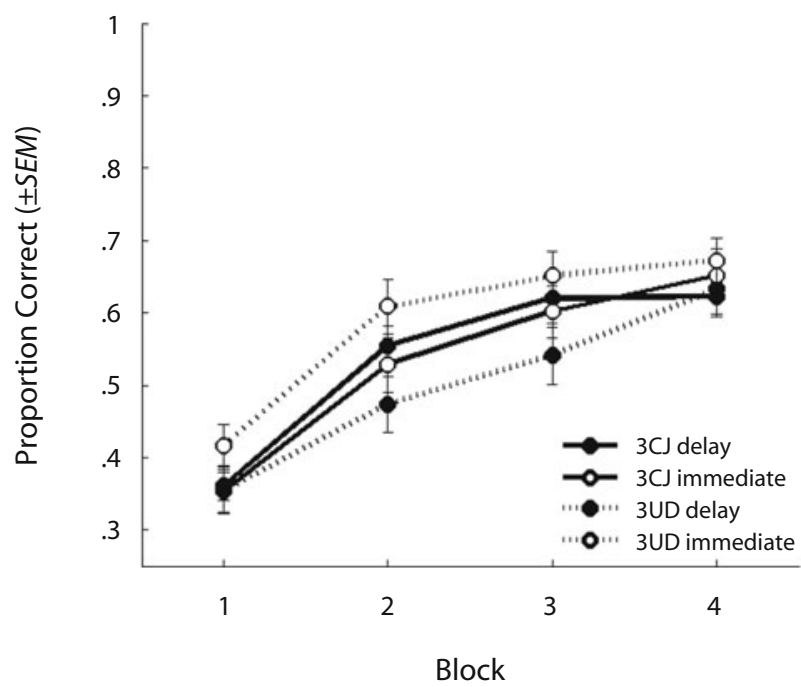

Figure 5. Proportion correct for the delayed- and immediatefeedback conditions of Experiment 2. The three-unidimensional condition (3UD) data from Experiment 1 have been replotted for reference. $3 \mathrm{CJ}$, three-decision-criteria conjunction condition. the hypothesis that distributing the criteria across multiple dimensions increases the availability of working memory resources that can be used to overcome the effect of drift resulting from criterial noise.

\section{EXPERIMENT 3}

The data from Experiments 1 and 2 are consistent with the hypothesis that working memory can be used to slow the drift rate of the decision criteria. This interpretation, however, is based on the claim that working memory demand is greater in the $3 \mathrm{UD}$ categories than in the $3 \mathrm{CJ}$ categories. To test this hypothesis directly, in Experiment 3, we used a dual-task procedure as a probe for working memory demand during the learning of the 3UD and 3CJ categories (Maddox, Ashby, et al., 2004; Zeithamova \& Maddox, 2007).

On each trial in Experiment 3, the participant categorized, received $500 \mathrm{msec}$ of feedback, and then completed a single four-item memory-scanning task. The idea is that performance of the memory-scanning task will prevent normal feedback processing and, consequently, will impair accuracy in categorization tasks with high working memory demand. Indeed, the memory-scanning task has been shown to be diagnostic of working memory demand in categorization tasks (Maddox, Ashby, et al., 2004; Zeithamova \& Maddox, 2007) - a result that is consistent with dual-task paradigms in which the secondary task, stimulus presentation, categorization response, and feedback are all coincident (e.g., Waldron \& Ashby, 2001; Zeithamova \& Maddox, 2006). Importantly, the degree to which the memory-scanning task will interfere with category learning should be directly proportional to the working memory demand associated with learning the task. Therefore, if the 3UD task taxes working memory to a greater extent than does the $3 \mathrm{CJ}$ task, categorization accuracy should be lower in the 3UD than in the 3CJ categories while the participants perform the memoryscanning task.

\section{Method}

\section{Participants and Design}

Fifty participants ( 25 in each task) were solicited from the University of Texas community and received course credit for participation. No participant completed more than one experimental condition. All of the participants were tested for 20/20 vision using a Snellen eye chart. Each participant completed one session with a duration of approximately $60 \mathrm{~min}$.

\section{Stimuli and Stimulus Generation}

Category learning. The category-learning stimuli were identical to those outlined in Experiment 1.

Memory scanning. On each trial, four digits were sampled randomly (without replacement) from the set of single-digit numbers (0-9). The four selected digits were displayed for $500 \mathrm{msec}$ in $48-$ point type in a horizontal array, each separated by 100 pixels and vertically centered on the screen. A blank screen was then displayed for 1,000 msec. Next, a single digit was sampled randomly, with .5 probability of being sampled from the memory set. The selected digit was displayed centered on the screen along with the question, "Was this item in the memory set?" The observer then responded yes 
or no by pressing one of two keys, which were different from those used for categorization.

\section{Procedure}

The procedure was identical to that in Experiment 1, with the following exceptions. For memory scanning, the participants were informed that high levels of performance were possible and that they should respond as quickly and accurately as possible. If performances in the memory-scanning task were below $90 \%$ accuracy at the end of any trial, the participants were told to increase their memory-scanning accuracy. These notifications stopped once memory-scanning accuracy was above $90 \%$. For category learning, there was no longer a delay between the response and feedback, and no mask was presented.

\section{Results and Theoretical Analyses Accuracy-Based Analyses}

Memory scanning. The participants performed the memory-scanning task with high accuracy, achieving an overall accuracy level of $96.9 \%$. There was no significant difference in memory-scanning accuracy across the 3UD $(96.6 \%)$ and 3 CJ $(97.1 \%)$ tasks $[t(48)=0.84, p>.40]$. Mean correct response time in the memory-scanning task was $1,434 \mathrm{msec}$. There was no significant difference in memory-scanning mean response times across the 3UD $(M=1,453 \mathrm{msec})$ and $3 \mathrm{CJ}(M=1,415 \mathrm{msec})$ tasks $[t(48)=0.73, p>.50]$.

Category learning. Recall that we successfully equated 3UD and 3CJ immediate-feedback performances in Experiment 2 . Thus, we can directly compare $3 \mathrm{UD}$ and $3 \mathrm{CJ}$ performances to determine which category structure is more difficult to learn when the feedback is followed immediately by a working-memory-demanding task and, by extension, which task places a greater demand on working memory during learning. Inspection of the average accuracy rates in Figure 6 suggests that there was a greater accuracy cost in the 3UD task than in the 3CJ task. A 2 (categorization task: 3 UD vs. $3 \mathrm{CJ}) \times 4$ (block) mixed design ANOVA was conducted on the accuracy rates to confirm this observation. The main effects of task $[F(1,48)=$ $\left.5.48, M S_{\mathrm{e}}=.064, p<.05\right]$ and block $[F(3,144)=38.70$, $\left.M S_{\mathrm{e}}=.008, p<.001\right]$ were significant, and the interaction $\left[F(3,144)=1.68, M S_{\mathrm{e}}=.008, p=.173\right]$ was nonsignificant. The main effect of task suggested that 3UD category learning (36\%) was significantly worse than $3 \mathrm{CJ}$ category learning $(45 \%) .{ }^{3}$ Consistent with the multidimensional benefit in absolute identification, these results support the claim that the working memory demand in the multidimensional task (3CJ) is reduced relative to the unidimensional task (3UD). Importantly, these data provide strong support for the hypothesis that the effect of drift is moderated by working memory demand.

\section{DISCUSSION}

In this article, we have reported the results from three experiments in which we tested the hypothesis that, in rule-based category-learning tasks, the negative impact of drift in the representation of the decision criteria and stimuli due to internal noise can be overcome by working memory resources. To make the effect of drift observable,

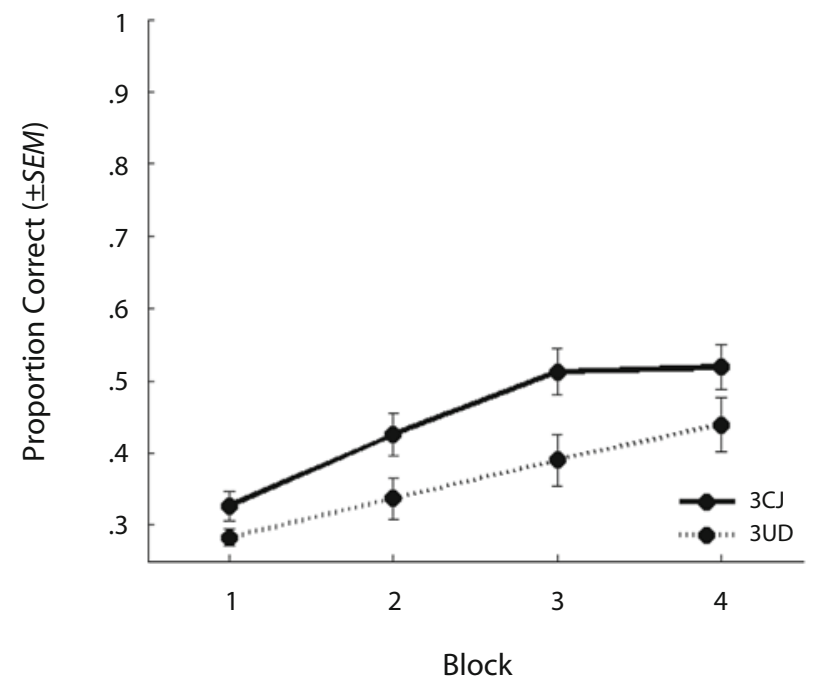

Figure 6. Proportion correct for the three-decision-criteria conjunction (3CJ) and three-unidimensional (3UD) conditions from Experiment 3.

we inserted a 5-sec delay between the categorization response and the delivery of corrective feedback. In Experiment 1 , drift during the delay impaired accuracy, but only when the demands on working memory resources associated with maintaining and manipulating multiple decision criteria were high. Furthermore, the model-based analyses suggest that this impairment was driven by poor learning of the placement of the decision criteria and a slight increase in noise associated with the representation of the decision criteria and stimulus. In Experiment 2, building on a classic finding in the absolute identification literaturenamely, that performance benefits by increasing the number of dimensions relevant to solving the task (Attneave, 1959; Garner, 1962; Miller, 1956; Pollack, 1952)—we demonstrated that distributing the decision criteria across multiple dimensions decreases the impact of drift during the delay. Consistent with the hypothesis that the multidimensional benefit observed in Experiment 2 was due to an increase in available working memory resources, we showed that working memory demand was lower in the multidimensional task. Taken together, these results suggest that rule-based category learning is robust to drift in the decision criteria as long as the available working memory resources are sufficient to slow the drift rate.

\section{The Multidimensional Benefit}

The interpretation of Experiment 2 depends on the extent to which results from absolute identification generalize to category learning. Claiming that there is a link between identification and categorization is not novel; several researchers have argued that the decision processes in identification and categorization are similar (Ashby \& Lee, 1991; Maddox, 2001; Nosofsky, 1986; Shepard et al., 1961). To our knowledge, however, this is the first report to show that the multidimensional benefit observed in absolute identification generalizes to rule-based category learning. We argue that the multidimensional benefit 
shown in Experiment 2 is driven by a reduction in working memory demand. To be clear, the classic interpretation is that the multidimensional benefit in absolute identification is driven by increased information transmission rather than by a decrease in memory demand (Miller, 1956). We speculate, however, that one by-product of increased information transmission would be a reduced demand on working memory resources. The results of Experiment 3, showing that the multidimensional rule-based task has a lower working memory demand than does the unidimensional rule-based task, support our conjecture.

How exactly is information transmission increased? To our knowledge, there are no data in the categorization literature that speak directly to this question. Of course, there are a number of ways in which efficiencies in information transmission could be achieved. In multidimensional, rule-based categorization tasks, it has been argued that independent decisions are made on the relevant stimulus dimensions (Ashby \& Gott, 1988). It may also be the case that efficiencies are gained by recoding the multidimensional information as a unidimensional decision variable, similar to accounts of visual detection and discrimination across multiple stimulus dimensions (Gorea, Caetta, \& Sagi, 2005; Gorea \& Sagi, 2000; J. P. Thomas \& Olzak, 1996).

\section{Theoretical Implications}

In previous work, delayed feedback has been shown to have no effect on learning in one-criterion, unidimensional (Maddox et al., 2003) and two-criteria, multidimensional (Maddox \& Ing, 2005) rule-based categorization tasks. Interestingly, both studies showed an effect of delayed feedback on information-integration category-learning tasks. Information-integration categories are typically generated by rotating unidimensional, rule-based categories $45^{\circ}$, such that optimal performance requires the integration of spatial frequency and orientation rather than parsing the stimulus space via decision criteria (Ashby et al., 1998; Ashby \& Ell, 2001). In contrast to the explicit processing that is assumed to mediate learning in rule-based tasks, learning is assumed to develop implicitly, involving the incremental acquisition of associations between stimuli and category responses in information-integration tasks (e.g., Ashby \& Waldron, 1999).

The delayed-feedback results on information-integration tasks are predicted by COVIS (competition between verbal and implicit systems; Ashby et al., 1998) - a biologically plausible, multiple-systems model of category learning. COVIS assumes that learning in rule-based tasks is dominated by an explicit hypothesis-testing system that depends on working memory and executive attention to learn decision criteria. Feedback serves to modify the relative strength of competing decision criteria on a trial-by-trial basis. Learning in information-integration tasks, however, is assumed to be dominated by an implicit procedural-based learning system that depends on a close temporal correspondence among stimulus, response, and feedback in order to strengthen the appropriate (stimulus-category) associations (Ashby et al., 1998). Delaying feedback disrupts this correspondence and impairs learning in informationintegration tasks. In contrast to rule-based tasks, the effect of delayed feedback should not depend on the working memory demand imposed by stabilizing drift in the decision criteria and, instead, should be observed in all informationintegration category-learning tasks. To test this prediction, we ran information-integration (II) analogues of the 2UD and $3 \mathrm{UD}$ categories (i.e., the Figure $1 \mathrm{~B}$ and $1 \mathrm{C}$ categories rotated $45^{\circ}$ counterclockwise $)$ in the delayed- $\left(n_{2 \mathrm{II}}=21\right.$, $\left.n_{3 \mathrm{II}}=21\right)$ and immediate-feedback $\left(n_{2 \mathrm{II}}=23, n_{3 \mathrm{II}}=18\right)$ conditions. In support of COVIS, the accuracy cost due to delaying feedback (averaged across participants and blocks) was statistically significant in the $2 \mathrm{II}[M=9 \%, F(1,42)=$ $\left.5.05, M S_{\mathrm{e}}=.077, p<.05\right]$ and $3 \mathrm{II}[M=13 \%, F(1,37)=$ $\left.14.42, M S_{\mathrm{e}}=.047, p<.001\right]$ conditions.

At first glance, it would seem that the results from the rule-based tasks are inconsistent with COVIS. Recall, however, that learning in the hypothesis-testing system is assumed to be highly dependent on working memory. Thus, COVIS predicts that learning should be impaired in rule-based tasks if the working memory demand is increased. The results from this study suggest that the working memory demands of the system were exceeded in the 3UD condition with a 5-sec feedback delay.

Conversely, the ability of COVIS to predict the impact of increased working memory demand due to increased drift in rule-based categorization is debatable. In previous computational implementations of COVIS, the effect of increased working memory demand due to the addition of a secondary task has been modeled by decreasing the likelihood that the hypothesis-testing system is able to successfully implement a newly selected decision criterion (Ashby \& Ell, 2002; Waldron \& Ashby, 2001). This approach could reasonably be used to model the effect of drift in the present experiments, because, as was previously mentioned, feedback is assumed to play a role in the selection of new decision criteria. This approach, however, is not general enough to account for the wide range of conditions in which drift in the decision criteria would be expected to impair rule-based category learning. Perhaps a more plausible alternative is to assume that the representation of the stimulus and the criteria are noisy and modeled by a zero-mean diffusion process (e.g., Ashby, 2000; Ratcliff, 1978). Thus, as feedback is delayed, the criterion representation will naturally drift away from its mean position and slow the learning rate. Working memory and attentional resources can be invoked to slow the drift rate, but as working memory demand increases, the hypothesis-testing system is less able to accomplish this goal. Past computational implementations of COVIS have not modeled the representation of decision criteria as a dynamic process. Such issues should receive consideration in future computational implementations of COVIS.

\section{Related Approaches in the Study of Visual Discrimination Learning}

Arguably, Gabor patterns have been the stimuli of choice for the study of visual discrimination learning. 
Despite the similar stimuli, there are a number of important differences between the research conducted on visual discrimination and the present experiments. For example, our emphasis is on characterizing the learning of categories comprised of Gabor filters rather than the discrimination between Gabor filters. That being said, it could be argued that the decision process in the present rule-based category-learning tasks is similar to the decision process in simultaneous visual discrimination tasks in the sense that both assume a comparison between, for example, the spatial frequency representation of the current stimulus and some other spatial frequency representation (or, in a signal detection framework, the comparison would be made on an arbitrary decision axis; R. D. Thomas, 1996). As we increase the number of decision criteria (from the 2UD categories to the 3 UD categories), we are increasing the number of simultaneous discriminations that need to be made. Making multiple simultaneous discriminations on a single stimulus dimension (i.e., spatial frequency) reduces performance in simultaneous discrimination tasks (Magnussen \& Greenlee, 1997; J. P. Thomas, Magnussen, \& Greenlee, 2000); thus, one would expect that accuracy for the 3UD categories would be lower than that for the 2UD categories in the immediate-feedback condition. The categories were constructed, however, such that accuracy in the immediate-feedback conditions was equated, thereby alleviating this potential task-difficulty confound. Given that task difficulty is equated, it is unclear how the data from simultaneous discrimination tasks would predict the interaction between delayed feedback and the number of decision criteria.

Moreover, in most visual discrimination tasks, there is no category structure. One of the novel contributions of our results is that the category structure is a critical element in predicting the impact of drift on internal representations. For example, changing the category structure by distributing the decision criteria across three dimensions (i.e., the 3CJ categories of Experiments 2 and 3) eliminates the effect of delayed feedback (Experiment 2). Thus, although there are important similarities, our results could not be anticipated on the basis of research from visual discrimination tasks.

The design of the present tasks (i.e., repeated exposure of stimuli with similar perceptual representations) would encourage perceptual learning, where the improvement in performance due to repeated stimulus exposure has been argued to be driven primarily by information external to the participant (Gibson, 1969) and mediated by relatively low-level mechanisms (e.g., Dosher \& Lu, 1999). The mechanisms involved in perceptual and category learning likely overlap. For instance, learning may depend on changes in both perceptual and criterial representations (Wenger, Copeland, Bittner, \& Thomas, 2008). In addition, trial-by-trial feedback is not necessary for perceptual learning (e.g., Herzog \& Fahle, 1997). Although this is also true for rule-based tasks with a single decision criterion (Ashby, Queller, \& Berretty, 1999), omitting feedback with the 3UD categories would likely impair accuracy, given the results of delaying feedback. Recent studies of perceptual learning suggest that feedback may be related to both en- hanced (Petrov, Dosher, \& Lu, 2006) and impaired (Eckstein, Abbey, Pham, \& Shimozaki, 2004) performances. Thus, it may be the case that delaying feedback would impact perceptual learning as well. It should be noted, however, that comparisons between perceptual learning and category learning may depend critically on the structure of the categories. For example, Casale and Ashby (2008) found evidence for the perceptual learning of categories constructed from dot patterns (Posner \& Keele, 1968), but only when the participants learned to distinguish between a category and random distortions of the category prototype (as opposed to two categories of dot patterns).

\section{Limitations and Future Directions}

The interpretation of these data focuses on the assumption of variability in the representation of the decision criteria (i.e., criterial noise). This assumption is supported by a wealth of empirical and theoretical data and has been a common component of computational models of rulebased category learning and decision making (Ashby, 1992a, 2000; Ashby et al., 1998; Ashby \& Lee, 1993; Bogacz, Brown, Moehlis, Holmes, \& Cohen, 2006; Busemeyer \& Townsend, 1993; Dorfman et al., 1975; Erev, 1998; Maddox \& Ashby, 1993; Mueller \& Weidemann, 2008; Treisman \& Williams, 1984). It is also common to assume that there is variability in the representation of the stimulus (e.g., Ashby \& Townsend, 1986; Durlach \& Braida, 1969; Green \& Swets, 1966). Given that the effects of delayed feedback are mediated by increasing the number and manipulating the distribution of decision criteria, it seems reasonable to speculate that our results are mediated by criterial noise. In the categorization tasks reported in this article, however, it is difficult to dissociate the effects of perceptual and criterial noise. For example, replicating the simulations shown in Figure 2 while assuming that there is drift in the representation of the stimulus, rather than in the representation of the decision criteria, does not change the qualitative pattern of predicted results. Future research will take advantage of paradigms that have been developed to dissociate the relative contributions of perceptual and criterial noise to category learning (e.g., Maddox, 2001).

The interpretation also assumes that the differential effect of delayed feedback across the categories is driven by an increase in the impact of criterial noise due to an increase in the number of decision criteria on a single stimulus dimension. In Experiments 1 and 2, a mask (a Gabor filter) was presented following the categorization response (i.e., prior to the delay). Numerous studies have shown that masks interfere with the representation of spatial frequency and orientation in visual discrimination tasks (Bennett \& Cortese, 1996; Lalonde \& Chaudhuri, 2002; Magnussen, Greenlee, Asplund, \& Dyrnes, 1991; Zhou, Kahana, \& Sekuler, 2004). Thus, the mask may have increased perceptual and/or criterial noise by interfering with the representation of the stimulus and/or decision criterion. Critically, however, the spatial frequency and orientation of the mask was selected at random on every trial, and this procedure was identical across the category structures. Therefore, although the mask may 
have increased perceptual and/or criterial noise, the mask cannot explain the differential effects of delayed feedback on the $2 \mathrm{UD}, 3 \mathrm{UD}$, and $3 \mathrm{CJ}$ categories.

The present data suggest that delayed feedback can affect rule-based category learning, but only when the working memory demand associated with holding a criterion representation in memory during the delay period exceeds working memory capacity. We found that the need to learn three decision criteria along the spatial frequency dimension was enough to slow learning when feedback was delayed. It is important to be clear that we are not arguing that there is something magical about three decision criteria. In fact, we would argue that there are a number of factors that will influence when delayed feedback will affect rule-based category learning. For example, the nature of the stimulus dimensions might also have an effect. Spatial frequency and orientation are separable (Garner, 1974; Maddox, 1992; Shepard, 1964) in the sense that they are processed more independently than integral dimension stimuli. Decision criterion learning might show a larger delayed-feedback effect if processing of the dimensions overlaps. Finally, the number of irrelevant dimensions present in the stimulus might also have an effect. An increase in the number of irrelevant dimensions that the participant must learn to ignore would likely tax working memory and degrade the perceptual representation of the stimulus and criteria.

These data suggest a number of important avenues for future empirical work. For example, in the present study, the effect of drift was made observable by fixing the feedback delay duration and manipulating the number of decision criteria. Alternatively, the effect of drift would also be observable if delay duration was varied and the number of criteria was fixed. For information-integration tasks, delaying feedback by as little as $2.5 \mathrm{sec}$ impairs accuracy (Maddox et al., 2003). The magnitude of the accuracy impairment should not increase as the delay duration increases beyond $2.5 \mathrm{sec}$, because the close temporal correspondence among stimulus, response, and feedback has already been sufficiently disrupted. In contrast, for rule-based tasks, the magnitude of the accuracy impairment should increase monotonically with delay duration, because the representation of the decision criterion would drift because of criterial noise. Thus, we would predict a crossover interaction for information-integration and rule-based tasks as the delay duration is increased from $2.5 \mathrm{sec}$. Clearly, the present (and future) empirical work will provide important constraints on the development of theories of categorization.

Another avenue for future research would be to examine the effects of extended training. The present data are mute on whether the effect of delayed feedback for the 3UD categories would persist with extended training. Accuracy in the 3UD delayed-feedback condition would likely increase with additional training, but we would also expect accuracy to increase in the 3UD immediate-feedback condition. This is an important issue for future work, but this unresolved issue does not negate the importance of the present results, which inform our understanding of the relationship among criterial learning, criterial noise, and working memory in rule-based tasks.

\section{Conclusions}

The results of these three experiments suggest that, under normal conditions, working memory resources can be invoked to slow drift in the decision criteria that would be expected, given the common assumption of a noisy representation of the decision criteria. Working memory, however, is critical for maintaining and manipulating decision criteria during the course of learning. As the number of decision criteria increases (at least along a single dimension), the available working memory resources are insufficient to slow the drift rate, and rule-based categorization accuracy is impaired.

The results of these experiments also represent an important integration of the absolute identification and categorization literatures. Moreover, these results challenge the previously held notion that rule-based tasks are not susceptible to the effect of delayed feedback. Instead, we demonstrated that the working memory demand associated with the number of dimensions relevant to solving the task moderates the effect of delayed feedback in rulebased categorization.

A thorough understanding of the impact of criterial noise on drift in decision criteria will have an impact beyond that of rule-based category learning. For instance, criterial noise has been central in the study of visual detection (Treisman \& Faulkner, 1985; Treisman \& Williams, 1984), discrimination, and identification (e.g., Maddox, 2001; Miller, 1956), as well as that of social categorization (e.g., Hall \& Crisp, 2005). Thus, given the intense interest in the contributions of perceptual and criterial noise among researchers in various fields, this research will serve as a basis for integrating the respective literatures.

\section{AUTHOR NOTE}

This research was supported in part by Grant NS047884 to S.W.E. and Grants MH59196 and MH077708 to W.T.M. from the National Institutes of Health, and by the Center for Perceptual Systems at the University of Texas, Austin. We thank Kelli Hejl, Scott Lauritzen, and Mina WilcoxGhanoonparvar for help with data collection. We also thank Joshua Solomon and three anonymous reviewers for providing helpful comments on an earlier version of the manuscript. Correspondence concerning this article should be addressed to S. W. Ell, Psychology Department, University of Maine, 5742 Little Hall, Room 301, Orono, ME 04469-5742 (e-mail: shawn.ell@umit.maine.edu) or W. T. Maddox, Department of Psychology, University of Texas, 1 University Station A8000, Austin, TX 78712 (e-mail: maddox@psy.utexas.edu).

\section{REFERENCES}

Akaike, H. (1974). A new look at the statistical model identification. IEEE Transactions on Automatic Control, 19, 716-723.

Allen, S. W., \& Brooks, L. R. (1991). Specializing the operation of an explicit rule. Journal of Experimental Psychology: General, 120, 3-19.

Ashby, F. G. (1992a). Multidimensional models of categorization. In F. G. Ashby (Ed.), Multidimensional models of perception and cognition (pp. 449-483). Hillsdale, NJ: Erlbaum.

Asнвy, F. G. (1992b). Multivariate probability distributions. In F. G. Ashby (Ed.), Multidimensional models of perception and cognition (pp. 1-34). Hillsdale, NJ: Erlbaum.

Astby, F. G. (2000). A stochastic version of general recognition theory. Journal of Mathematical Psychology, 44, 310-329.

Ashby, F. G., Alfonso-Reese, L. A., Turken, A. U., \& Waldron, 
E. M. (1998). A neuropsychological theory of multiple systems in category learning. Psychological Review, 105, 442-481.

Ashby, F. G., \& ELL, S. W. (2001). The neurobiology of human category learning. Trends in Cognitive Sciences, 5, 204-210.

Ashby, F. G., \& ELL, S. W. (2002). Single versus multiple systems of category learning: Reply to Nosofsky and Kruschke (2002). Psychonomic Bulletin \& Review, 9, 175-180.

Ashby, F. G., \& GotT, R. E. (1988). Decision rules in the perception and categorization of multidimensional stimuli. Journal of Experimental Psychology: Learning, Memory, \& Cognition, 14, 33-53.

Ashby, F. G., \& Lee, W. W. (1991). Predicting similarity and categorization from identification. Journal of Experimental Psychology: General, 120, 150-172.

Ashby, F. G., \& Lee, W. W. (1993). Perceptual variability as a fundamental axiom of perceptual science. In S. C. Masin (Ed.), Foundations of perceptual theory (pp. 369-399). Amsterdam: Elsevier.

Ashby, F. G., Queller, S., \& Berretty, P. M. (1999). On the dominance of unidimensional rules in unsupervised categorization. Perception \& Psychophysics, 61, 1178-1199.

Ashby, F. G., \& Townsend, J. T. (1986). Varieties of perceptual independence. Psychological Review, 93, 154-179.

AshBy, F. G., \& WALDRON, E. M. (1999). On the nature of implicit categorization. Psychonomic Bulletin \& Review, 6, 363-378.

ATtNeAVE, F. (1959). Applications of information theory to psychology: A summary of basic concepts, methods, and results. New York: Holt, Rinehart \& Winston.

Benjamin, A. S., Diaz, M., \& Wee, S. (2009). Signal detection with criterion noise: Applications to recognition memory. Psychological Review, 116, 84-115.

Bennett, P. J., \& CoRtese, F. (1996). Masking of spatial frequency in visual memory depends on distal, not retinal, frequency. Vision Research, 36, 233-238.

Bogacz, R., Brown, E., Moehlis, J., Holmes, P., \& Cohen, J. D. (2006). The physics of optimal decision making: A formal analysis of models of performance in two-alternative forced-choice tasks. Psychological Review, 113, 700-765.

BRAINARD, D. H. (1997). Psychophysics software for use with MATLAB. Spatial Vision, 10, 433-436.

Busemeyer, J. R., \& Myung, I. J. (1992). An adaptive approach to human decision making: Learning theory, decision theory, and human performance. Journal of Experimental Psychology: General, 121, 177-194.

Busemeyer, J. R., \& Townsend, J. T. (1993). Decision field theory: A dynamic-cognitive approach to decision making in an uncertain environment. Psychological Review, 100, 432-459.

Casale, M. B., \& Ashby, F. G. (2008). A role for the perceptual representation memory system in category learning. Perception \& Psychophysics, 70, 983-999.

Cox, D. R., \& Miller, H. D. (1965). The theory of stochastic processes. London: Methuen.

Dorfman, D. D., Saslow, C. F., \& Simpson, J. C. (1975). Learning models for a continuum of sensory states reexamined. Journal of Mathematical Psychology, 12, 178-211.

Dosher, B. A., \& LU, Z. L. (1999). Mechanisms of perceptual learning. Vision Research, 39, 3197-3221.

DurLaCh, N. I., \& BraidA, L. D. (1969). Intensity perception: I. Preliminary theory of intensity resolution. Journal of the Acoustical Society of America, 46, 372-383.

Eckstein, M. P., Abbey, C. K., Pham, B. T., \& Shimozaki, S. S. (2004). Perceptual learning through optimization of attentional weighting: Human versus optimal Bayesian learner. Journal of Vision, 4 (12, art. 3), 1006-1019. doi:10.1167/4.12.3

EREv, I. (1998). Signal detection by human observers: A cutoff reinforcement learning model of categorization decisions under uncertainty. Psychological Review, 105, 280-298.

Erickson, M. A., \& KruschKe, J. K. (1998). Rules and exemplars in category learning. Journal of Experimental Psychology: General, 127, 107-140.

Folstein, J. R., \& Van Petten, C. (2004). Multidimensional rule, unidimensional rule, and similarity strategies in categorization: Eventrelated brain potential correlates. Journal of Experimental Psychology: Learning, Memory, \& Cognition, 30, 1026-1044.
GARNER, W. R. (1962). Uncertainty and structure as psychological concepts. London: Wiley.

GARNER, W. R. (1974). The processing of information and structure. New York: Wiley.

Gibson, E. J. (1969). Principles of perceptual learning and development. New York: Appleton-Century-Crofts.

Gorea, A., Caetta, F., \& SAgi, D. (2005). Criteria interactions across visual attributes. Vision Research, 45, 2523-2532.

Gorea, A., \& SAGI, D. (2000). Failure to handle more than one internal representation in visual detection tasks. Proceedings of the National Academy of Sciences, 97, 12380-12384.

Green, D. M., \& Swets, J. A. (1966). Signal detection theory and psychophysics. New York: Wiley.

HALL, N. R., \& CRISP, R. J. (2005). Considering multiple criteria for social categorization can reduce intergroup bias. Personality \& Social Psychology Bulletin, 31, 1435-1444.

Herzog, M. H., \& FAHLE, M. (1997). The role of feedback in learning a vernier discrimination task. Vision Research, 37, 2133-2141.

KAC, M. (1962). A note on learning signal detection. IRE Transactions on Information Theory, IT-8, 126-128.

Kubovy, M., \& Healy, A. F. (1977). The decision rule in probabilistic categorization: What it is and how it is learned. Journal of Experimental Psychology: General, 106, 427-446.

LALONDE, J., \& CHAUdHURI, A. (2002). Task-dependent transfer of perceptual to memory representations during delayed spatial frequency discrimination. Vision Research, 42, 1759-1769.

Maddox, W. T. (1992). Perceptual and decisional separability. In F. G. Ashby (Ed.), Multidimensional models of perception and cognition (pp. 147-180). Hillsdale, NJ: Erlbaum.

MADDOX, W. T. (2001). Separating perceptual processes from decisional processes in identification and categorization. Perception \& Psychophysics, 63, 1183-1200.

Maddox, W. T. (2002). Toward a unified theory of decision criterion learning in perceptual categorization. Journal of the Experimental Analysis of Behavior, 78, 567-595.

Maddox, W. T., \& Ashby, F. G. (1993). Comparing decision bound and exemplar models of categorization. Perception \& Psychophysics, 53, 49-70.

Maddox, W. T., Ashby, F. G., \& Bohil, C. J. (2003). Delayed feedback effects on rule-based and information-integration category learning. Journal of Experimental Psychology: Learning, Memory, \& Cognition, 29, 650-662.

Maddox, W. T., Ashby, F. G., Ing, A. D., \& Pickering, A. D. (2004). Disrupting feedback processing interferes with rule-based but not information integration category learning. Memory \& Cognition, 32, $582-591$.

Maddox, W. T., Filoteo, J. V., HeJl, K. D., \& Ing, A. D. (2004). Category number impacts rule-based but not information-integration category learning: Further evidence for dissociable category learning systems. Journal of Experimental Psychology: Learning, Memory, \& Cognition, 30, 227-235.

Maddox, W. T., \& ING, A. D. (2005). Delayed feedback disrupts the procedural-learning system but not the hypothesis-testing system in perceptual category learning. Journal of Experimental Psychology: Learning, Memory, \& Cognition, 31, 100-107.

Magnussen, S., \& GreEnLeE, M. W. (1997). Competition and sharing of processing resources in visual discrimination. Journal of Experimental Psychology: Human Perception \& Performance, 23, 16031616.

Magnussen, S., Greenlee, M. W., Asplund, R., \& Dyrnes, S. (1991). Stimulus-specific mechanisms of visual short-term memory. Vision Research, 31, 1213-1219.

Miller, G. A. (1956). The magical number seven plus or minus two: Some limits on our capacity for processing information. Psychological Review, 63, 81-97.

Mueller, S. T., \& Weidemann, C. T. (2008). Decision noise: An explanation for observed violations of signal detection theory. Psychonomic Bulletin \& Review, 15, 465-494.

NosOFsKy, R. M. (1986). Attention, similarity, and the identification categorization relationship. Journal of Experimental Psychology: General, 115, 39-57.

Nosofsky, R. M., Palmeri, T. J., \& McKinley, S. C. (1994). Rule- 
plus-exception model of classification learning. Psychological Review, 101, 53-79.

Pelli, D. G. (1997). The Video Toolbox software for visual psychophysics: Transforming numbers into movies. Spatial Vision, 10, 437-442.

Petrov, A. A., Dosher, B. A., \& Lu, Z.-L. (2006). Perceptual learning without feedback in non-stationary contexts: Data and model. Vision Research, 46, 3177-3197.

PollaCK, I. (1952). The information of elementary auditory displays. Journal of the Acoustical Society of America, 24, 745-749.

Posner, M. I., \& Keele, S. W. (1968). On the genesis of abstract ideas. Journal of Experimental Psychology, 77, 353-363.

Ratcliff, R. (1978). A theory of memory retrieval. Psychological Review, 85, 59-108.

Regehr, G., \& Brooks, L. R. (1995). Category organization in free classification: The organizing effect of an array of stimuli. Journal of Experimental Psychology: Learning, Memory, \& Cognition, 21, 347-363.

SHEPARD, R. N. (1964). Attention and the metric structure of the stimulus space. Journal of Mathematical Psychology, 1, 54-87.

Shepard, R. N., Hovland, C. I., \& Jenkins, H. M. (1961). Learning and memorization of classifications. Psychological Monographs, 75, $1-42$.

Takane, Y., \& Shibayama, T. (1992). Structures in stimulus identification data. In F. G. Ashby (Ed.), Multidimensional models of perception and cognition (pp. 335-362). Hillsdale, NJ: Erlbaum.

Thomas, E. A. C. (1973). On a class of additive learning models: Error correcting and probability matching. Journal of Mathematical Psychology, 10, 241-264.

Thomas, J. P., Magnussen, S., \& Greenlee, M. W. (2000). What limits simultaneous discrimination accuracy? Vision Research, 40, 31693172 .

Thomas, J. P., \& Olzak, L. A. (1996). Uncertainty experiments support the roles of second-order mechanisms in spatial frequency and orientation discriminations. Journal of the Optical Society of America, 13, 689-696.

Thomas, R. D. (1996). Separability and independence of dimensions within the same-different judgment task. Journal of Mathematical Psychology, 40, 318-341.

Treisman, M., \& FaulKner, A. (1985). Can decision criteria inter- change locations? Some positive evidence. Journal of Experimental Psychology: Human Perception \& Performance, 11, 187-208.

Treisman, M., \& Williams, T. C. (1984). A theory of criterion setting with an application to sequential dependencies. Psychological Review, 91, 68-111.

WALDRON, E. M., \& Ashby, F. G. (2001). The effects of concurrent task interference on category learning: Evidence for multiple category learning systems. Psychonomic Bulletin \& Review, 8, 168-176.

Wenger, M. J., Copeland, A. M., Bittner, J. L., \& Thomas, R. D. (2008). Evidence for criterion shifts in visual perceptual learning: Data and implications. Perception \& Psychophysics, 70, 1248-1273.

WickENs, T. D. (1982). Models for behavior: Stochastic processes in psychology. San Francisco: Freeman.

Zeithamova, D., \& Maddox, W. T. (2006). Dual-task interference in perceptual category learning. Memory \& Cognition, 34, 387-398.

Zeithamova, D., \& Maddox, W. T. (2007). The role of visuospatial and verbal working memory in perceptual category learning. Memory \& Cognition, 35, 1380-1398.

Zhou, F., Kahana, M. J., \& Sekuler, R. (2004). Short-term episodic memory for visual textures: A roving probe gathers some memory. Psychological Science, 15, 112-118.

\section{NOTES}

1. Attentional processes are likely involved as well (Ashby et al., 1998). For simplicity, however, we use the phrase working memory to refer to both working memory and attentional processes throughout the article.

2. Percentages of responses accounted for were again high-ranging, from $70 \%-72 \%$ for the hypothesis-testing models when applied to the immediate- and delayed-feedback conditions.

3. Recall that although there was no statistically significant difference between immediate-feedback accuracy scores in the 3UD and 3CJ tasks (see Experiment 2), accuracy in the 3UD task was numerically higher. Similar to the pattern of results in Experiment 2, the working memory manipulation in Experiment 3 interfered with the easier 3UD task, thereby ruling out task difficulty as a plausible alternative hypothesis.

(Manuscript received December 8, 2008; revision accepted for publication March 20, 2009.) 